\title{
The salivary gland salivation stimulating peptide from Locusta migratoria (Lom-SG-SASP) is not a typical neuropeptide
}

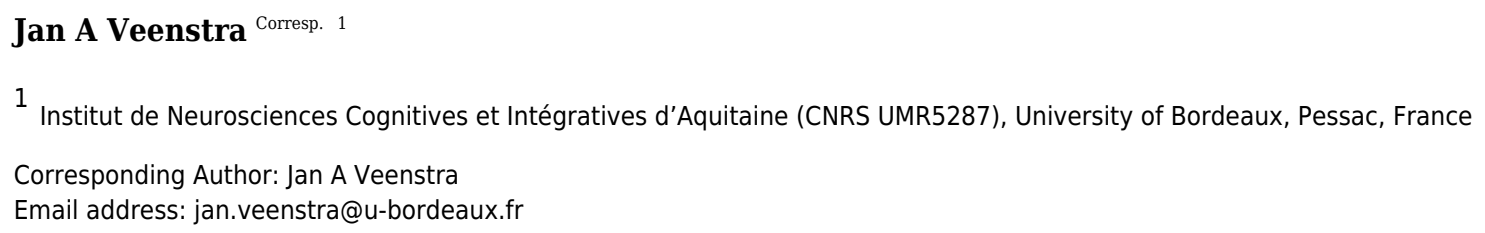

The salivary gland salivation stimulating peptide was identified from the salivary glands of the migratory locust by its ability to stimulate CAMP production in the same tissue. The gene coding for this peptide has recently been identified and been shown to code for a precursor consisting of a signal peptide, several copies of the peptide separated by LysArg doublets and a few other peptides. These data are consistent with it being a neuropeptide. However, antiserum raised to this peptide labels the acini of the salivary glands while RT-PCR only gives positive results in the salivary gland, but not in any ganglion of the central nervous system. Thus, this peptide is not a typical neuropeptide as previously assumed. 
1 The salivary gland salivation stimulating peptide from Locusta migratoria (Lom-SG-SASP) is

2 not a typical neuropeptide

3

4 Jan A. Veenstra

5

6

7 INCIA, UMR 5287 CNRS, Université de Bordeaux, Pessac, France

12 Corresponding Author:

13 Jan Veenstra, INCIA UMR 5287 CNRS, Université de Bordeaux, allée Geoffroy St Hillaire, CS

14 50023, 33615 Pessac Cedex, France

15 Email address: jan.veenstra@u-bordeaux.fr

16

17

18

19 
20 Abstract

21

The salivary gland salivation stimulating peptide was identified from the salivary glands

23 of the migratory locust by its ability to stimulate cAMP production in the same tissue. The gene

24 coding for this peptide has recently been identified and been shown to code for a precursor

25 consisting of a signal peptide, several copies of the peptide separated by Lys-Arg doublets and a

26 few other peptides. These data are consistent with it being a neuropeptide. However, antiserum

27 raised to this peptide labels the acini of the salivary glands while RT-PCR only gives positive

28 results in the salivary gland, but not in any ganglion of the central nervous system. Thus, this

29 peptide is not a typical neuropeptide as previously assumed.

30

31

32

33

34

35

36 Key words : SG-SASP ; PC1 ; PC2 ; RT-PCR ; antiserum ; immunohistology 


\section{Introduction}

Insect neuropeptides are interesting for two different reasons. On the one hand, insects are protostomes, while vertebrates are deuterostomes. Thus, comparing insect with vertebrate neuropeptide regulatory systems provides insight as to how structures and functions may have changed during evolution. On the other hand, many insect species are pests and vectors of disease and thus responsible for serious agricultural damage as well as the transmission of human disease. As insects are becoming increasingly resistant to classical pesticides novel insecticides are constantly needed and it is has repeatedly been suggested that agonists or antagonists of insect neuropeptide receptors might offer a solution (e.g. Audsley et al., 2015 ; van Hiel et al., 2010). Inhibition or at least disruption of feeding by insects would be very attractive as it would presumably avoid or diminish economic damage, or in the case of disease vectors, might reduce transmission of infectious agents. As production of saliva is usually a first and necessary step in feeding the regulation of salivation by neuropeptides is particularly interesting.

A pentadecapeptide was isolated from the salivary glands of the migratory locust by its ability to stimulate the production of cAMP in the same glands at concentration of $10^{-6} \mathrm{M}$ (Veelaert et al., 1995). As it also stimulates salivation (Veelaert et al., 1995), its characteristics suggests it to be neuropeptide that likely acts as a neurotransmitter rather than a hormone. If it were a hormone, one would expect it to stimulate the production of cAMP and salivation in the nanomolar, rather than in the micromolar range and it would be expected in a neurhemal organ, rather than in the salivary gland itself (Veelaert et al., 1995). Unlike most insect neuropeptides orthologs of this peptide have not been identified from any other arthropod, suggesting that it may not be universally present in insects. This might be advantageous as any pesticide based on it could be relatively selective. The genome sequence combined with the RNAseq data of the migratory locust (Wang et al., 2014) made it possible to predict a likely precursor for this peptide (Veenstra, 2014). There is single amino acid mismatch between the predicted precursor and the identified peptide, but the peptide sequence is bounded by putative convertase cleavage sites (Fig. 1) and it is thus highly likely that this is indeed the precursor of this peptide. The precursor has all the hallmarks of a typical neuropeptide precursor: a signal peptide and a propeptide encoding multiple copies of the peptide separated by Lys-Arg convertase cleavage sites. It thus appeared of interest to study this putative neuropeptide in more detail, but as reported here the 
68 peptide turned out not to be a typical neuropeptide, in spite of the data suggesting otherwise.

69

70

71

72

\section{Materials and methods}

\subsection{Locusts.}

Adult and fifth instar Locusta migratoria were purchased at a local pet store. They were kept for five to seven days at $25{ }^{\circ} \mathrm{C}$ and fed fresh grass once a day before being used. Tissues were dissected under saline and either frozen immediately at $-80^{\circ} \mathrm{C}$ for subsequent RNA extraction, or used for immunohistology.

\subsection{Immunohistology}

I choose the SVTVREVGDLFQEWLQGNVN sequence for making antisera, as this sequence is present in multiple copies on the precursor (Fig. 1). Two mg (purity $84 \%$, Proteogenix, Schiltigheim, France) were conjugated to $5 \mathrm{mg}$ of bovine serum albumin using difluorodinitrobenzeze as the conjugation reagent as documented by Tager (Tager, 1976). Polyclonal mouse antisera were raised in three six week-old NMRI female mice as described previously (Veenstra and Ida, 2014). Tissues were fixed for 1 to $2 \mathrm{hrs}$ at room temperature. All other immunohistological procedures are the same as described (Veenstra and Ida, 2014). Primary antiserum was diluted 1:2,000, the secondary antiserum, DyLight-488-conjugated goat anti-mouse IgG that was from Jackson ImmunoResearch Europe (Newmarket, Suffolk, UK), $1: 1,000$.

\subsection{Bioinformatics.}

cDNA equences coding for the L. migratoria orthologs of vertebrate $\mathrm{PC} 1$ and $\mathrm{PC} 2$ are not present in the databases and were therefore obtained by using a combination of the published genome sequence and Trinity on sequences extracted from the various short read archives (SRAs) for this species available at NCBI (SRR014351, SRR014352, SRR058432, SRR058446, SRR058447, SRR058448, SRR058449, SRR058450, SRR058451, SRR058452, SRR058453, SRR058454, SRR058455, SRR058456, SRR058457, SRR058458, SRR058488, SRR058489, SRR058490, SRR058491, SRR058492, SRR058493, SRR058494, SRR058495, SRR058496, SRR058497, SRR058498, SRR058499, SRR058500, SRR058501, SRR058502, SRR058503, 
99

100

101

102

103

104

105

106

107

108

109

110

111

112

113

114

115

116

117

118

119

120

121

122

123

124

125

126

127

128

129

SRR1032161, SRR1032192, SRR167712, SRR513208, SRR513209, SRR513210 and

SRR513211) using methodology described in detail elsewhere (Veenstra, 2016). Protein and cDNA sequences for L. migratoria $\mathrm{PC} 1$ and $\mathrm{PC} 2$ are provided in the supplementary data.

\subsection{RT-PCR}

The following tissues were dissected : brain, suboeosphageal ganglion, pro- and mesothoracic ganglia combined, the meta-thoracic ganglia combined with all abdominal ganglia, salivary gland, fat body and midgut. For the analysis of the expression of PC1 and PC2 Malpighian tubules and flight muscle were also analyzed. At least two samples were processed completely independently for each of these tissues. Each sample containing tissues from at least four different animals. From salivary glands, fatbody, Malpighian tubules and flight muscle, small parts were taken from four different animals. For the midgut four longitudinal half midguts were processed individually. RNA extraction was performed using mini spin columns from Macherey-Nagel. Next RNA (800-1000 ng) was reverse transcribed in a $20 \mu 1$ reaction using Moloney Murine Leukemia Virus Reverse Transcriptase (New England Biolabs, Evry, France) and random primers. One $\mu \mathrm{l}$ of the resulting cDNA was next amplified by PCR using OneTaq Quick-Load DNA Polymerase (New England Biolabs) with specific primers for each mRNA. Primers used are : for the salivary gland salivation stimulating peptide: 5 'GCCTTCCTGCTAGTCGTCTG-3 and 5'-TACCTTTTGCCCACTCTTGG-3'; for actin: 5'AGGGCTGTTTTTCCCTCAAT-3' and 5'-GAAGGTGTGGTGCCAGATTT -3'; for PC1: 5'ACAACCACGTGCACAAGAAG-3' and 5'-TGAATGCGACTAAGCCACAG -3' ; and for PC2: 5'-GGTGGACTACCTGGAACACG-3' and 5'-TGTGGATATTCTCCCCAGGT -3' . PCR profiles consisted of 90 second denaturing at $94^{\circ} \mathrm{C}$ followed by 32 cycles of 30 seconds at $94^{\circ} \mathrm{C}$, 15 seconds at the annealing temperature and 15 seconds at $68^{\circ} \mathrm{C}$ the amplification was followed by 5 min extension at $68^{\circ} \mathrm{C}$. Annealing temperatures were $55^{\circ} \mathrm{C}, 64^{\circ} \mathrm{C}, 64^{\circ} \mathrm{C}$ and $60^{\circ} \mathrm{C}$ for the salivary gland salivation stimulating peptide, actin, PC1 and PC2 respectively. Controls in which water replaced the cDNA showed no PCR amplification.

\section{Results and Discussion}

I anticipated that an antiserum would allow the identification of neurons expressing this 
130 peptide. However all three mice produced antisera that labeled the acinar cells of the salivary 131 glands (Fig. 2), while immunoreactive material was completely absent from the central nervous

132 system. Preimmune antisera from the same mice or diluted antisera that were absorbed with

133 SVTVREVGDLFQEWLQGNVN (over night, $10 \mu \mathrm{g} / \mathrm{ml}$ ) were not immunoreactive (Fig. 3), thus

134 showing that the observed immunoreactivity is specific. This suggested that the peptide was

135 made by the salivary gland itself, rather than by the nervous system. Intron spanning primers

136 were designed to look for expression of the gene coding the peptide and results revealed

137 amplification only in the salivary glands (Fig. 4). The PCR-amplified band was sequenced using

138 the primers for amplification and the sequencing results confirmed the expected sequence (Fig.

$139 \mathrm{~S} 1$ ). Thus there is independent confirmation that this gene is expressed in the salivary gland, but

140 neither in the central nervous system, the fatbody nor the midgut. RT-PCR confirmed strong

141 expression of this gene as (1) amplicons become visible after 20 cycles (Fig. 4) and (2) the

142 slightest contamination of thoracic ganglia with a small piece of salivary gland (these tissues are

143 closely associated with one another) leads to false positives. The integrity of the cDNA samples

144 were checked by looking for expression of actin as a control.

145 The Lys-Arg convertase cleavage sites in the precursor are identical to those commonly

146 found in neuropeptide precursors and thus suggested the presence of a neuropeptide specific

147 convertase in the salivary gland. The cDNA sequences of the two locust orthologs of vertebrate

148 PC1 and PC2 were obtained using Trinity on the various short read archives (SRAs) for this

149 species available at NCBI (Fig. S2) and intron spanning primers were designed to look for their

150 expression in the salivary gland. PC1 was found not only in the salivary gland, but also in other

151 peripheral tissues (Fig. 5), but PC2 was absent from the salivary glands. It thus appears that the

152 tissue distribution of PC1 in migratory locusts and perhaps other arthropods is much broader than

153 in vertebrates (Seidah et al, 2013).

154 A question that remains to be answered is what the function of this peptide might be.

155 Salivary glands typically secrete digestive enzymes in an exocrine fashion, but in principle, it

156 could also be secreted on the basolateral membrane into the hemolymph. Only the latter

157 possibility could explain how it could stimulate the salivary glands to produce cylic AMP. In

158 spite of this and the precedent for autocrine stimulation by dopamine in the tick salivary gland

159 (Koči, Šimo \& Park, 2014), there are several arguments to suggests that the peptide is secreted as 160 an exocrine rather than an autocrine or paracrine. First, the situation in ticks remains highly 
161 unusual. Secondly, in the tick dopamine is made only in a minor cell type, while SASP is made

162 in the major cell type. Thirdly, SASP is made as a protein precursor and as such should be

163 expected to be co-secreted with the digestive enzymes in the same granules (this is not the case

164 for dopamine, which is present in different granules). It would be very surprising if such

165 digestive enzyme containing granules would be secreted into the hemolymph. Fourth, although

166 dopamine in the tick salivary gland is present in relatively high concentrations, it remains a

167 minor product, while SASP as suggested by the immunochistological data as well as the RT-

168 PCR seems to be a major product of the salivary gland. Fifth, it is to be expected that the Lys-

169 Arg cleavage sites in the SASP precursor are cleaved efficiently, however for the peptide

170 identified by Veelaert et al. (1995) to be produced, this site needs to be still there, for a single

171 Arg residue is not easily cleaved by convertase. Hence, relatively small amounts of the active

172 peptide are expected to be made, while the likely much larger amounts of the peptide used here

173 as antigen, were not identified as a stimulator of cyclic AMP production. This seems to suggests

174 that the stimulation of cyclic AMP production is an accidental artifact, as perhaps also suggested

175 by the high concentrations needed of this peptide to do so.

176 Even if this may suggest an exocrine function for this peptide, it does not answer the

177 question what this function entails. As saliva is generally reabsorbed with the food, its tempting

178 to speculate that it somehow facilitates digestion or perhaps functions

179 In the last decade a large number of putative neuropeptide genes have been identified in

180 genome sequences of a wide variety of invertebrate species, sometimes based exclusively on the

181 presence of signal peptide and Lys-Arg cleavage sites that separate the presumptive

182 neuropeptides in the precursor. In many cases the predicted peptides show clear homology to

183 known neuropeptides, while in other cases they have subsequently been shown to activate G-

184 protein coupled receptors (e.g. Bauknecht et al., 2015). However, as illustrated here the presence

185 of a signal peptide, reputable convertase cleavages sites and multiple copies of the same or a

186 very similar peptide do not make a neuropeptide precursor.

187

188 4. References

189

190 N. Audsley, R.E. Down, G protein coupled receptors as targets for next generation pesticides.

191 Insect Biochem. Mol. Biol. 67 (2015) 27-37. doi: 10.1016/j.ibmb.2015.07.014. 
192

193 P. Bauknecht, G. Jékely, Large-scale combinatorial deorphanization of Platynereis neuropeptide

194 GPCRs. Cell Rep. 12 (2015) 684-693. doi.org/10.1016/j.celrep.2015.06.052.

195

196

J. Koči, L. Šimo, Y Park, Autocrine/paracrine dopamine in the salivary glands of the blacklegged

197

tick Ixodes scapularis. J. Insect Physiol. 62 (2014) 39-45.

198 doi.org/10.1016/j.jinsphys.2014.01.007.

199

200

N.G. Seidah, M.S. Sadr, M. Chrétien, M. Mbikay, The multifaceted proprotein convertases: their 201 unique, redundant, complementary, and opposite functions. J. Biol. Chem. 288 (2013) 21473202 21481. doi: 10.1074/jbc.R113.481549.

203

204

H.S. Tager, Coupling of peptides to albumin with difluorodinitrobenzene. Anal. Biochem. 71

205 (1976) 367-375.

206

207

M.B. van Hiel, T. van Loy, J. Poels, H.P. Vandersmissen, H. Verlinden, L. Badisco, J. Vanden Broeck, Neuropeptide receptors as possible targets for development of insect pest control agents. Adv. Exp. Med. Biol. 692 (2010) 211-226.

210

D.Veelaert, L. Schoofs, P. Proost, J. Van Damme, B. Devreese, J. Van Beeumen, A. De Loof,

212 Isolation and identification of Lom-SG-SASP, a salivation stimulating peptide from the salivary

213 glands of Locusta migratoria, Regul. Pept. 57 (1995) 221-226. doi.org/10.1016/0167-

214 0115(95)00035-A

215

216 J.A. Veenstra, Mono-and dibasic proteolytic cleavage sites in insect neuroendocrine peptide

217 precursors. Arch. Insect Biochem. Physiol. 43 (2000) 49-63. doi: 10.1002/(SICI)1520-

218 6327(200002)43:2<49::AID-ARCH1>3.0.CO;2-M.

219

220 J.A. Veenstra, The contribution of the genomes of a termite and a locust to our understanding of 221 insect neuropeptides and neurohormones. Front, Physiol. 5 (2014) 454. doi: 
224 J.A. Veenstra, Similarities between decapod and insect neuropeptidomes. PeerJ 26;4 (2016)

225 e2043. doi: 10.7717/peerj.2043.

226

227 J.A. Veenstra, T. Ida, More Drosophila enteroendocrine peptides: Orcokinin B and the

228 CCHamides 1 and 2. Cell Tissue Res.357 (2014) 607-621. doi: 10.1007/s00441-014-1880-2.

229

230 X. Wang, X. Fang, P. Yang, X. Jiang, F. Jiang, D. Zhao, B. Li, F. Cui, J. Wei, C. Ma, Y. Wang, 231 He J, Y. Luo, Z. Wang, X. Guo, W. Guo, X. Wang, Y. Zhang, M. Yang, S. Hao, B. Chen, Z. Ma, 232 D. Yu, Z. Xiong, Y. Zhu, D. Fan, L. Han, B. Wang, Y. Chen, J. Wang, L. Yang, W. Zhao, Y. 233 Feng, G. Chen, J. Lian, Q. Li, Z. Huang, X. Yao, N. Lv, G. Zhang, Y. Li, J. Wang, J. Wang, B. 234 Zhu, L. Kang, The locust genome provides insight into swarm formation and long-distance 235 flight, Nat. Commun. 5 (2014) 2957. doi: 10.1038/ncomms3957. 


\section{Figure 1}

Conceptual translation of the of the predicted open reading frame of SG-SASP from Locusta migratoria

Conceptual translation of the SASP mRNA predicted from transcriptome and genome sequences (Veenstra, 2016). Amino acid sequences highlighted in yellow indicate the signal peptide, Lys-Arg doublets highlighted in red are predicted convertase cleavage sites. In blue are the multiple copies of SVTVREVGDLFQEWLQGNVN, the major peptide encoded by the SASP gene. The amino acid sequence obtained by Veelaert et al. (1995) is not present in this sequence, but there is a single copy of EVGDLFEEWLQGNMN that is highlighted in black. The molecular mass of the EVGDLFEEWLQGNMN differs by less than 1 dalton from that of EVGDLFKEWLQGNMN. It seems plausible that an incorrect interpretation of the Edman degradation is responsible for the difference between the peptide sequence as experimentally determined and that deduced from the various DNA sequences. Similar minor differences were found for other Locusta neuropeptides (Veenstra, 2014).

EVGDLFEEWLQGNMN is predicted to be cleaved from its pecursor by typical neuropeptide convertases. The Lys-Arg doublet at its C-terminal in the precursor is the canonical neuropepide precursor cleavage site, while cleavage at the single Arg residue at its Nterminal is supported by the presence of another Arg residue at the - 6 position (Veenstra, 2000). The latter Arg residue is part of another Lys-Arg doublet, that is the preferred convertase substrate and should thus be expected to be cleaved rapidly on exposure of the precursor to convertase. Once this site has been cleaved, the single Arg residue can no longer be cleaved. Consequently, it must be expected that little of EVGDLFEEWLQGNMN will be produced as most of the time the Lys-Arg site will be processed preferentially and once this has occurred there is no longer an Arg residue in position -6 to support cleavage at the single Arg residue. Based on the precursor sequence and known convertase cleavage preferences in insects (Veenstra, 2000) one would expect it be processed mostly into 
SVTVREVGDLFQEWLQGNVN, the peptide used for raising an antiserum.

MALKTLAAFLLVVCLAQLTCASPAPAPNRRSTDESTADSSSATVTTSANNATS DADTISSNDTSSDDIFGEIGEKVEGFFGHLFGKRDIEIPSDVFTKLYQEWAKG RPSRSVTVRDTGKNFTVGDLFQEWLQGNVNKRSVTVREVGNLFQEWLQGNVNK RSVTVREVGDLFQEWLQGNVNKRSVTVREVGDLFQEWLQGNVNKRSVTVREVG DLFQEWLQGNVNKRSVTVREVGDLFQEWLQGNVNKRSVTVREVGDLFQEWLQG NVNKRSVTVREVGDLFQEWLQGNVNKRSVTVREVGDLFQEWLQGNVNKRSVTV REVGDLFQEWLQGNVNKRSVTVREVGDLFEEWLQGNMNKRSVTVRDTDNSSTM GDLIKEWLKGNLNERSVTVRDTGKNFTVGELFQEWLQGNVNKRSVTVRDTDNS STMNERSVTV 


\section{Figure 2}

Immunohistological localization of SG-SASP.

Immunoreactive salivary gland peptide in the adult salivary gland of $L$. migratoria. Note the strong labeling in the acinar cells. Scale bar $250 \mu \mathrm{m}$.

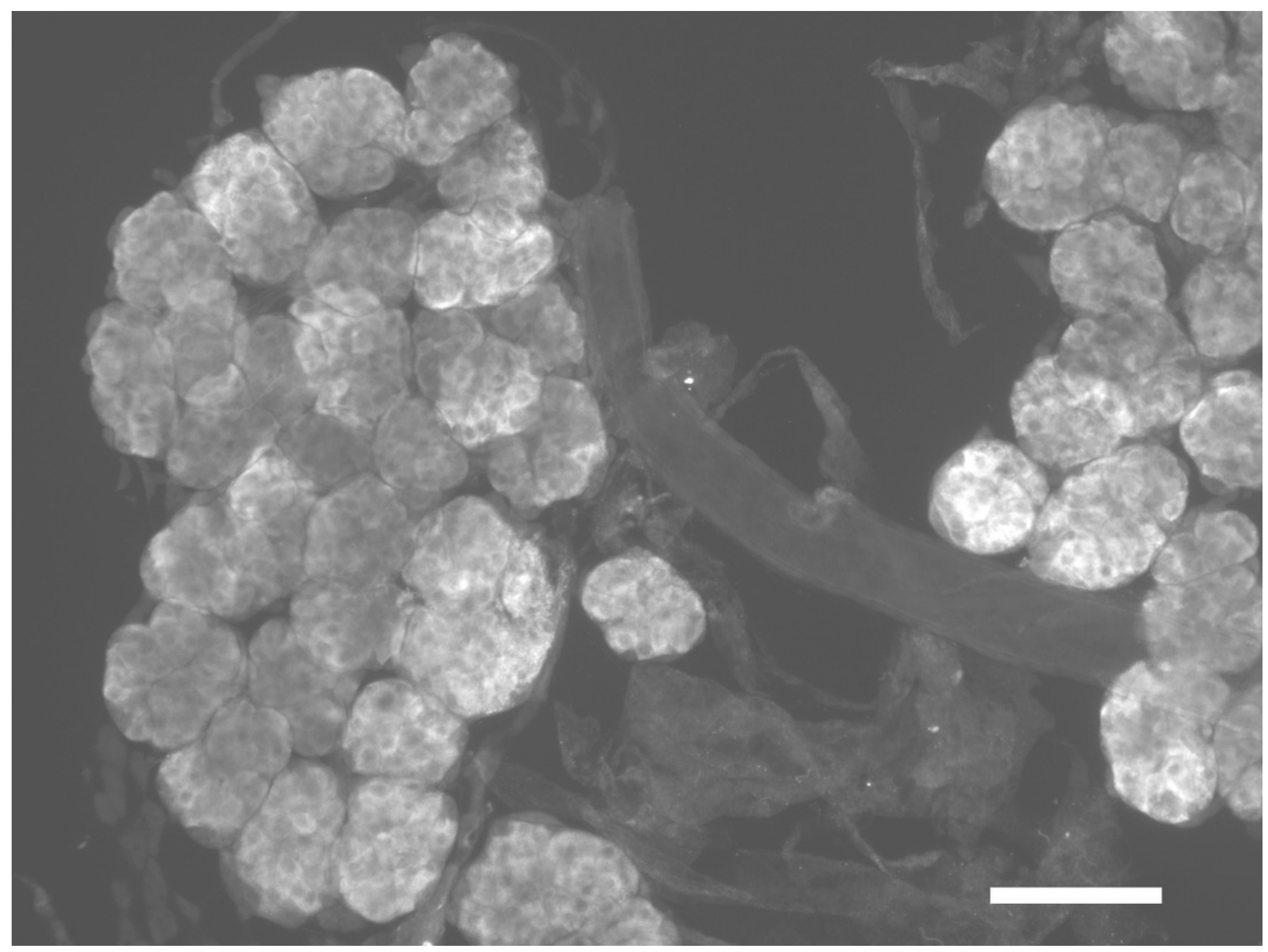




\section{Figure 3}

\section{Antiserum specificity}

Different pieces of the same salivary gland were exposed to pre-immune serum (a), immune serum (b), or immune serum preabsorbed with SVTVREVGDLFQEWLQGNVN. Note that no immunoreactivity is found in the preimmune serum and the immunoreactivity is abolished after preabsorption to SG-SASP.

*Note: Auto Gamma Correction was used for the image. This only affects the reviewing manuscript. See original source image if needed for review.
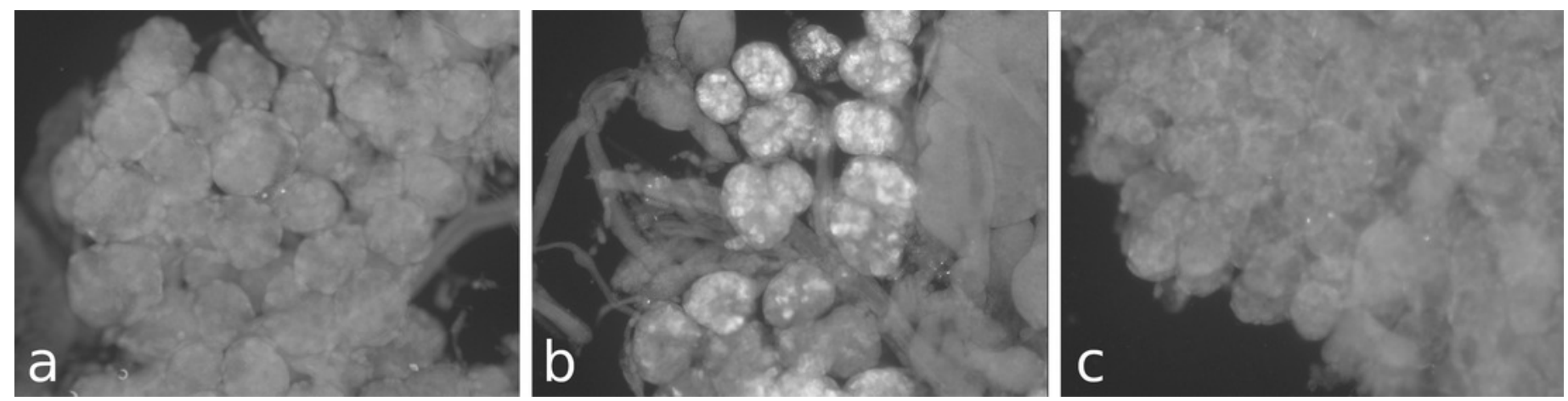


\section{Figure 4}

RT-PCR of the salivary gland peptide.

RT-PCR on different tissues from L. migratoria for the expression of SG-SASP and actin.

Numbers indicate the number of PCR cycles employed. Note that 20 cycles is sufficient to show expression in the salivary gland, while 35 cycles do not show any expression in the nervous system. TG $1 \& 2$, the pro- and meso-thoracic ganglion combined, TG3 \& AGs the methathoracic ganglion together with all the abdominal ganglia. Complete gels with markers are shown in the supplementary data.

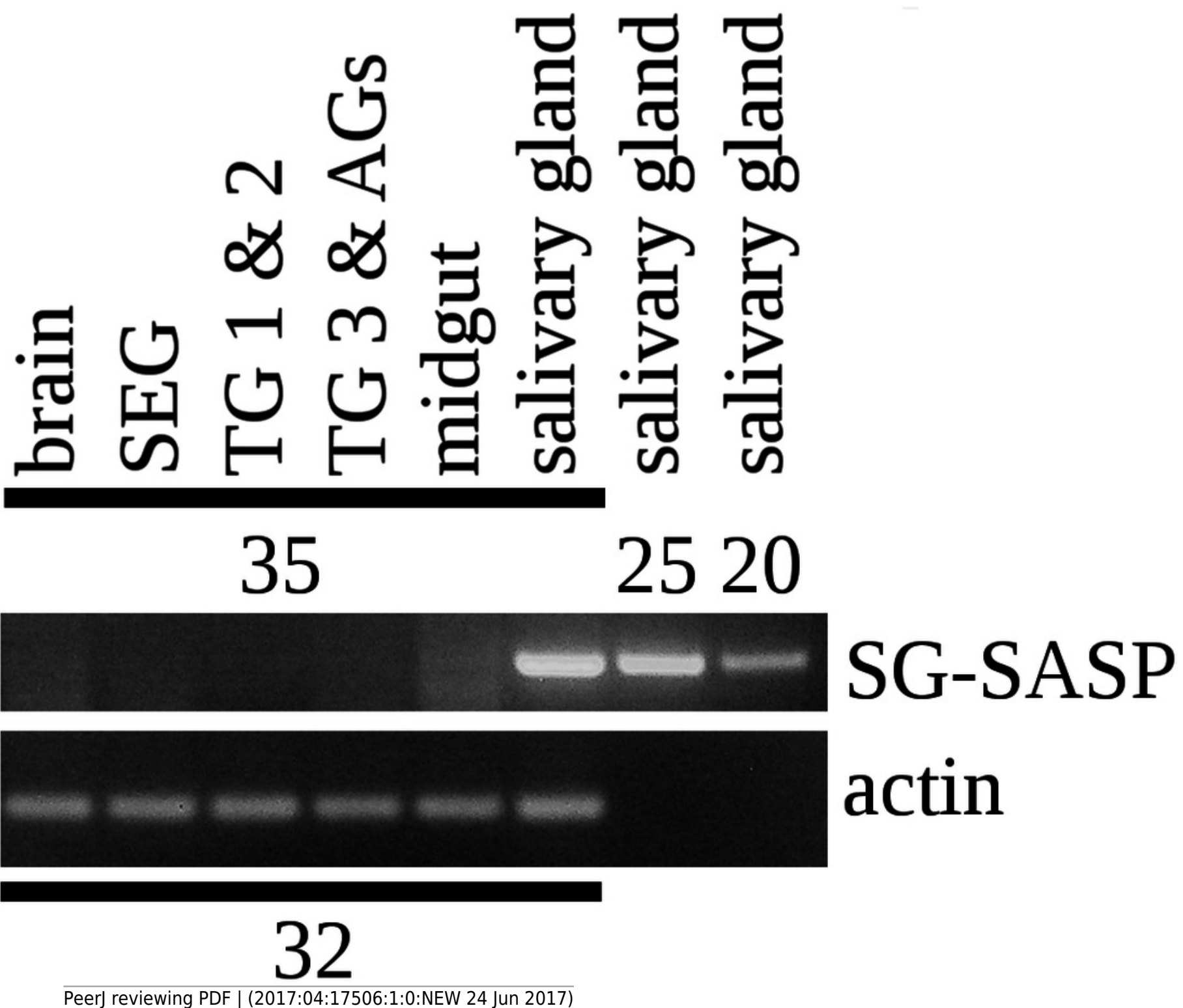




\section{Figure 5}

RT-PCR of PC1 and PC2.

RT-PCR on different tissues from L. migratoria for the expression of PC1 and PC2 convertaes with actin as a control. Note that PC1 is widely expressed, including in the salivary gland, while the expression of PC2 is much more limited and not expressed in the salivary gland. Thirty two cycles of PCR in each sample. Complete gels with markers are shown in the supplementary data. 

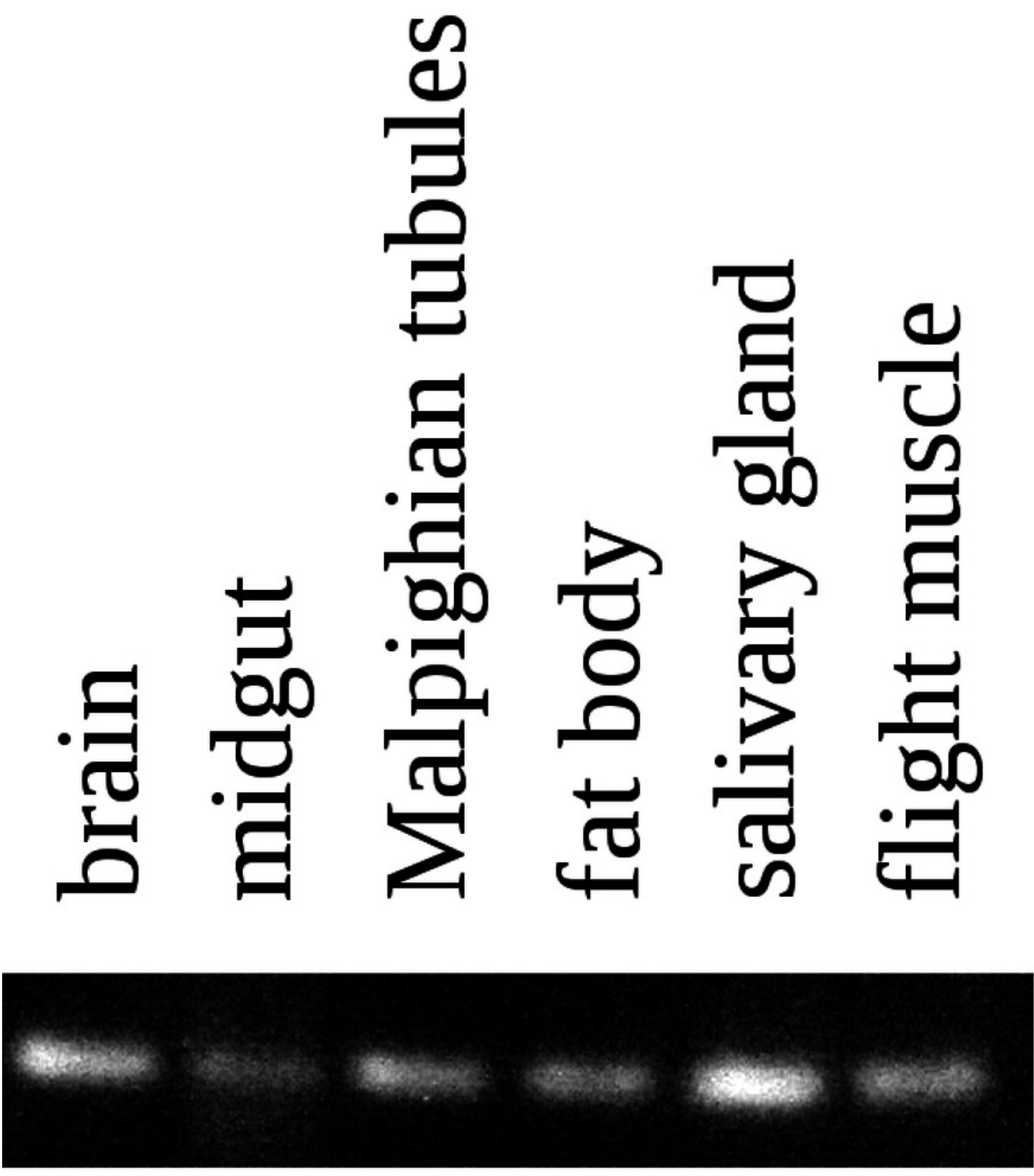

PC1

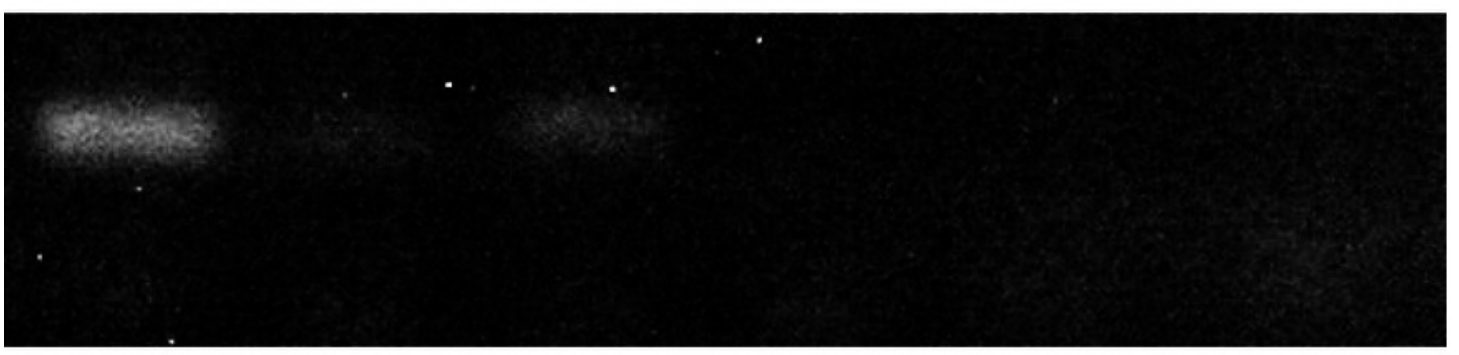

PC2

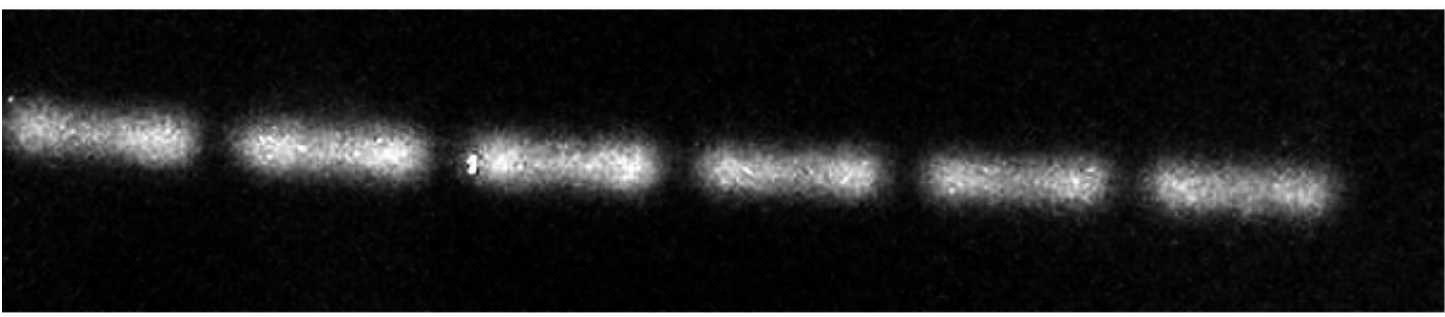

actin 